\title{
The Nature of the Local Group
}

\author{
Antonio Aparicio \\ University of La Laguna and Instituto de Astrofísica de Canarias, Via \\ Láctea, E-38205 La Laguna, Tenerife, Spain
}

\begin{abstract}
The Local Group provides an interesting and representative sample of galaxies in the rest of the Universe. The high accuracy with which many problems can be addressed in Local Group galaxies is of paramount importance for understanding galaxy formation and evolution. This contribution presents a short review of overall Local Group properties followed by short discussions of five topics in which the study of Local Group members provides particularly significant information. These topics are only examples of the usefulness and potential of Local Group research. The five selected topics are the formation of the Milky Way, galaxy destruction and tidal streams, detailed galactic chemical evolution, star formation history determination, and low surface brightness extended structures.
\end{abstract}

\section{Introduction}

The main motivation for studying the Local Group is that it is our nearest sample of galaxies. This would be a rather weak reason if we could not assume that the Local Group galaxies are a good, unbiased, representative sample of the galaxies in the rest of the universe. Only in such a case could the more accurate results that we can obtain for the Local Group members be extended to other galaxies. In fact, the Local Group contains members of most galaxy types: big design spirals (Andromeda, the Milky Way, M33); luminous irregulars (the Large and Small Magellanic Clouds); dim irregulars (NGC 6822, IC 1613, Leo A, etc.); a possible blue compact dwarf (IC 10); a nucleated dwarf elliptical (M32); dwarf ellipticals (NGC 205, NGC 147, NGC 185); dwarf spheroidals (Sculptor, Ursa Minor, etc.), and a highly stripped galaxy (Sagittarius $\mathrm{dSph}$ ). The only important types of galaxies not present in the Local Group are ellipticals (nondwarfs) and cDs (see van den Bergh 2000).

In this contribution, after an overview of Local Group properties, I will review a few general topics with a common characteristic: that the study of Local Group members should provide a significant improvement in our knowledge of the problem. The topics I have selected are the following:

- Galaxy formation: the Milky Way

- Galaxy destruction and tidal streams: the Sagittarius dSph galaxy

- Age-metallicity laws: detailed chemical evolution 


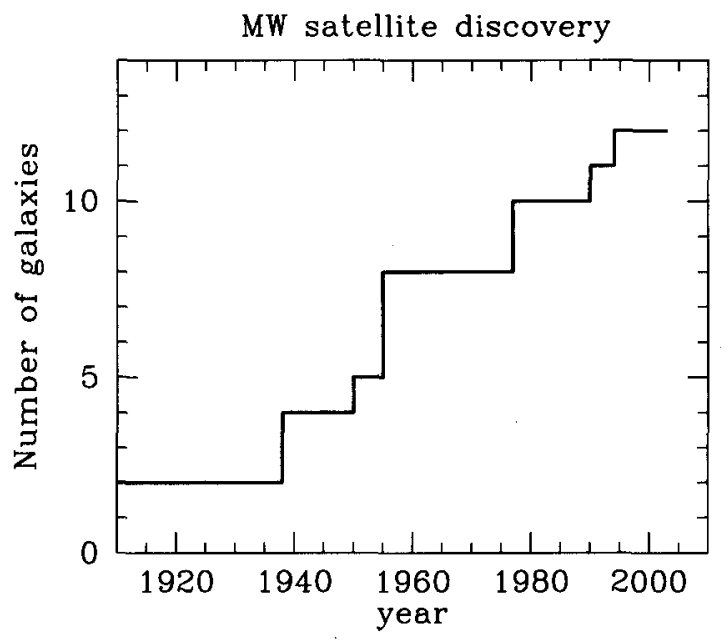

Figure 1. The Milky Way satellite system discovery. The number of known Milky Way satellites is plotted against the year. The plot seems to indicate that the discovering era is not yet finished.

- Quantitative determinations of star formation histories

- Extended low surface brightness structures in dwarf galaxies

The properties of the Local Group and its galaxies have recently been reviewed in several meetings (The Local Group: Comparative and Global Properties (Layden, Smith, \& Storm 1994); The Stellar Content of the Local Group Galaxies (Whitelock \& Cannon 1999) and in the Canary Islands Winter School Stellar Astrophysics for the Local Group (Aparicio, Herrero \& Sánchez 1998). Recent fundamental monographic reviews have been given by Grebel (1997), Mateo (1998), and van den Bergh (2000).

\section{The Local Group: An overview}

The number of Local Group galaxy members has been increasing since Hubble (1936) introduced the term "Local Group" itself. Hubble mentioned only ten galaxies as members of the Local Group: the Milky Way, the Large Magellanic Cloud (LMC), the Small Magellanic Cloud (SMC), Andromeda, M32, NGC 205, M33, NGC 6822, IC 1613, and IC 10 (which Hubble did not consider a secure member). Since then, galaxies have been discovered continuously and the rate of discover shows no sign of abating for the moment (see the enlightening figure 1.1 in van den Bergh 2000).

Deciding whether or not a galaxy is a Local Group member is not a straightforward task. Certainly the best criterion is dynamical: member galaxies should be gravitationally bound to the Local Group. But i) the mass of the Local Group is not reliably known and ii) the tangential velocity of galaxies cannot yet be 


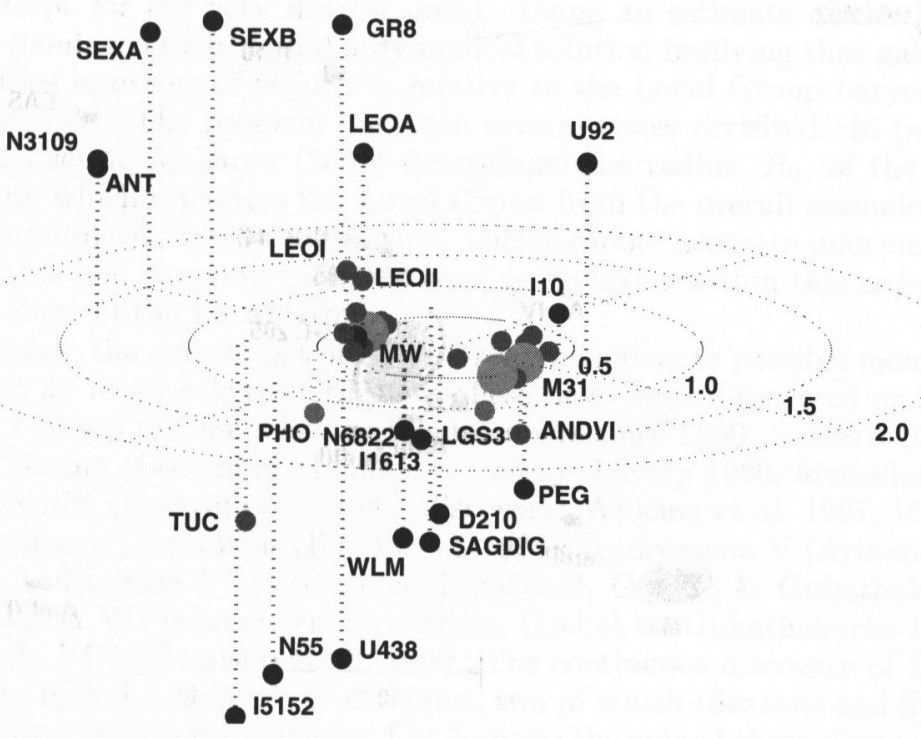

Figure 2. The Local Group in perspective. Labels on the concentric circles are distances to the Local Group barycenter in Mpc. The two galaxy subsystems around the Milky Way and Andromeda are visible.

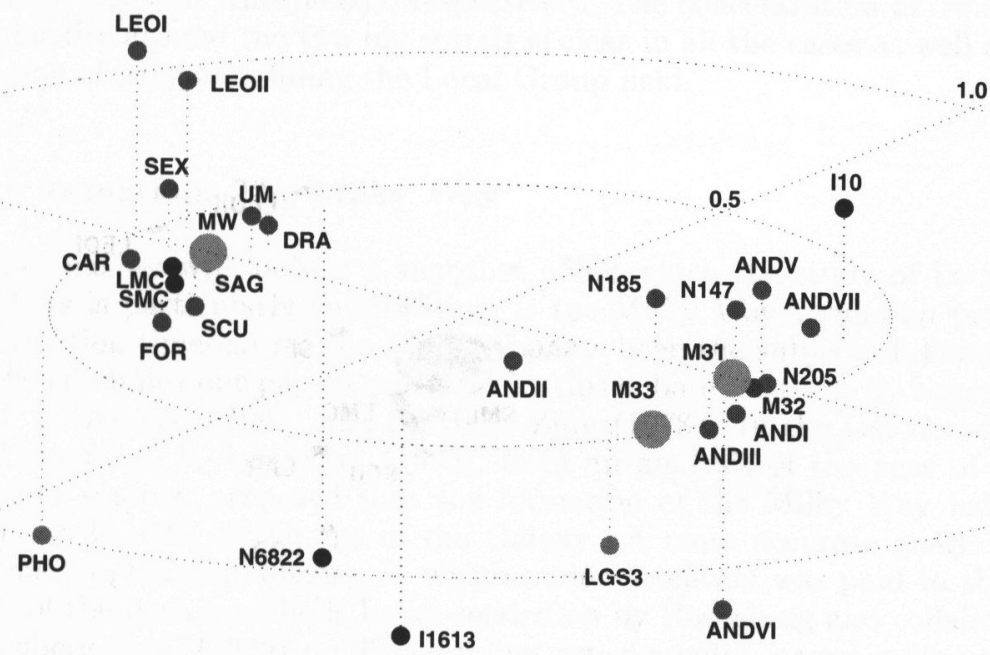

Figure 3. The central region of the Local Group in perspective showing in detail the Milky Way and Andromeda subsystems. Labels on the concentric circles are distances to the Local Group barycenter in Mpc. 
UGCA92

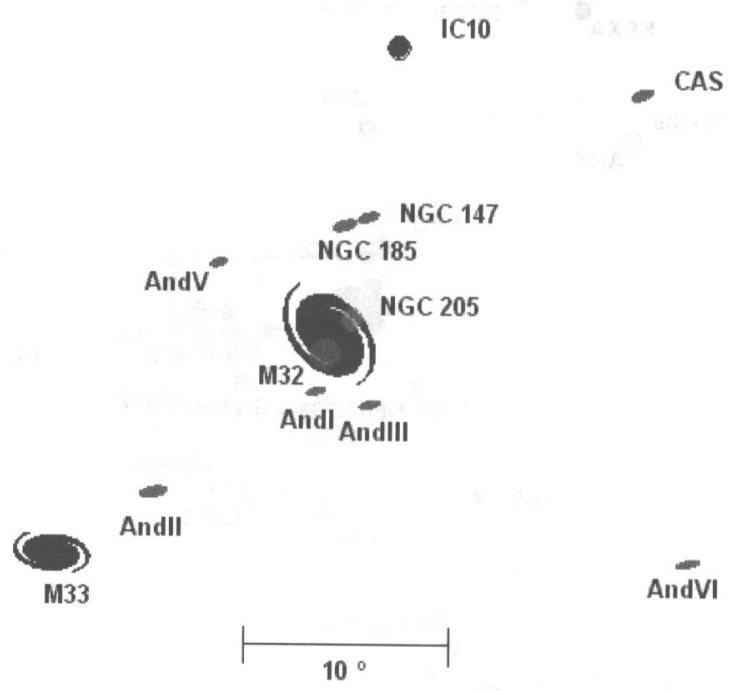

Figure 4. The Andromeda subsystem as seen from the Milky Way.
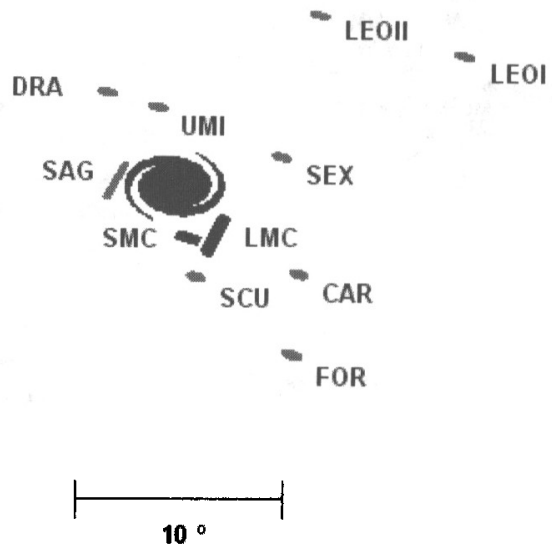

Figure 5. The Milky Way subsystem as seen from the Andromeda. 
measured (except for the very nearest ones). Using an estimate of the Local Group mass, Sandage (1986) found a dynamical solution implying that galaxies having velocities in excess of $60 \mathrm{~km} \mathrm{~s}^{-1}$ relative to the Local Group barycenter should be unbound. The problem has been several times revisited. In particular, Karachentsev \& Makarov (2001) determined the radius, $R_{0}$, of the zero velocity sphere, which separates the Local Group from the overall cosmological expansion, obtaining $R_{0}=0.96 \pm 0.05 \mathrm{Mpc}$. Lacking more accurate information, it is a reasonable and common choice to consider galaxies within this radius as probable members of the Local Group.

Summarizing, the current list of Local Group members or possible members includes about 35 to 40 galaxies. Eight of these have been discovered or identified as Local Group members or possible members since 1990: Sextans (Irwin et al. 1990); Tucana (Corwin et al. 1985, discovery; Lavery 1990, identification as member); Antlia (Corwin et al. 1985, discovery; Whiting et al. 1997, identification as member); Sagittarius (Ibata et al. 1994); Andromeda V (Armandroff et al. 1998); Andromeda VI (also called Pegasus II, Grebel, \& Guhathakurta 1999), Andromeda VII (also called Cassiopeia, Grebel, \& Guhathakurta 1999) and Andromeda VIII (Morrison et al. 2003). The continuous discovery of Local Group galaxies includes Milky Way satellites, two of which (Sextans and Sagittarius) were discovered in the nineties. Fig. 1 shows the rate of these discoveries. Nothing indicates that the discovery process is now finished.

Fig. 2 shows a general perspective of the Local Group. Fig. 3 is a zoom of the former showing the Milky Way and Andromeda subsystems in more detail. Figs 4 and 5 show the Andromeda and Milky Way subsystems as seen from the Milky Way and Andromeda, respectively. The concentration of dwarf spheroidal galaxies around the two big spirals is clear in all the cases as well as the distribution of irregulars filling the Local Group field.

\section{Galaxy formation: The Milky Way}

The first case that we will look at a snapshot of, in which the study of Local Group members is particularly enlightening, is the Milky Way formation process. The formation timescale of the Milky Way has been the subject of debate for years. The two milestone papers representing this debate are those by Eggen, Lynden-Bell, \& Sandage (1962) and Searle \& Zinn (1978). In the last decade Chaboyer, Demarque, \& Sarajedini (1996), from an analysis of the ages of a globular cluster sample, proposed that the formation of the Milky Way halo lasted $5 \mathrm{Gyr}$, or $30-40 \%$ of the age of the Galaxy. A more accurate analysis based on some 50 globular clusters, in which major attention was paid to the homogeneity of the data sample, is being carried on by Rosenberg and collaborators. Rosenberg et al. (1999) published a first set of results corresponding to the inner $20 \mathrm{kpc}$. A further extension of the work (Rosenberg et al. 2004) to the external halo $(40 \mathrm{kpc})$ indicates that perhaps two separate globular cluster populations are present, one of them $20 \%$ younger than the other (Fig. 6). Clusters are coeval (within the errors) in each group, the oldest one containing about $70 \%$ of the objects. Besides these two components a few still younger clusters exist. These are represented by only two objects in the Rosenberg et al. (2004) sample. 


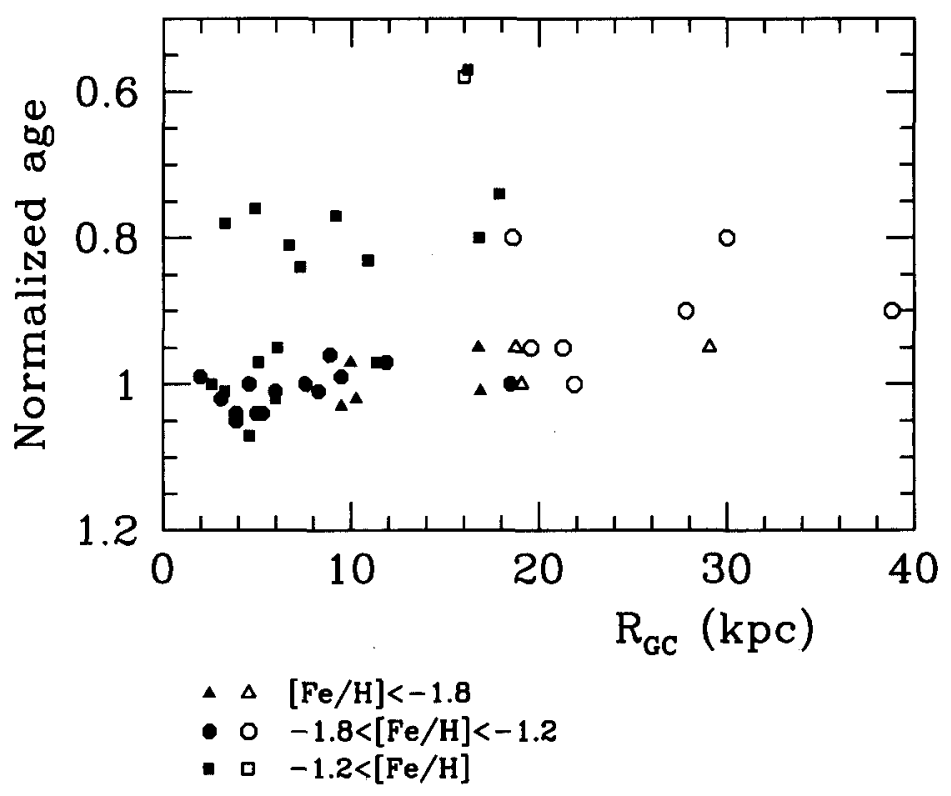

Figure 6. Age of Milky Way halo globular clusters as a function of galactocentric distance. Open dots correspond to an extended sample and are preliminary (Rosenberg et al. 2004).

\section{Galaxy destruction and tidal streams: The Sagittarius dSph}

The discovery of the Sagittarius dSph (Ibata et al. 1994) and of the fact that it is in an advanced state of tidal disruption have been of paramount relevance in the galaxy interaction research field. In particular, the Sagittarius dwarf is a living example of a fundamental process of galaxy evolution: the disruption and merging of dwarf galaxies in the potential well of giants, which is one of the predictions of the hierarchical galaxy formation scenario. The Sagittarius dwarf extends over a stream that completely enwraps the Milky Way in a polar orbit. The accuracy reached by several searches and positive and negative detections of the Sagittarius stream at different galactic latitudes is making it possible to develop an accurate orbital model for it and to constrain the Milky Way potential well. Fig. 7 shows the orbital model for the Sagittarius stream by Martínez-Delgado et al. (2003). The center of the galaxy marked in the figure corresponds to the globular cluster M54. Other globular clusters are also marked. One of them, Pal 12 , is particularly young.

\section{Age-Metallicity laws: Detailed chemical evolution}

A knowledge of the chemical enrichment process is fundamental to a proper understanding of the evolution of galaxies. In the simple closed-box model, it only 


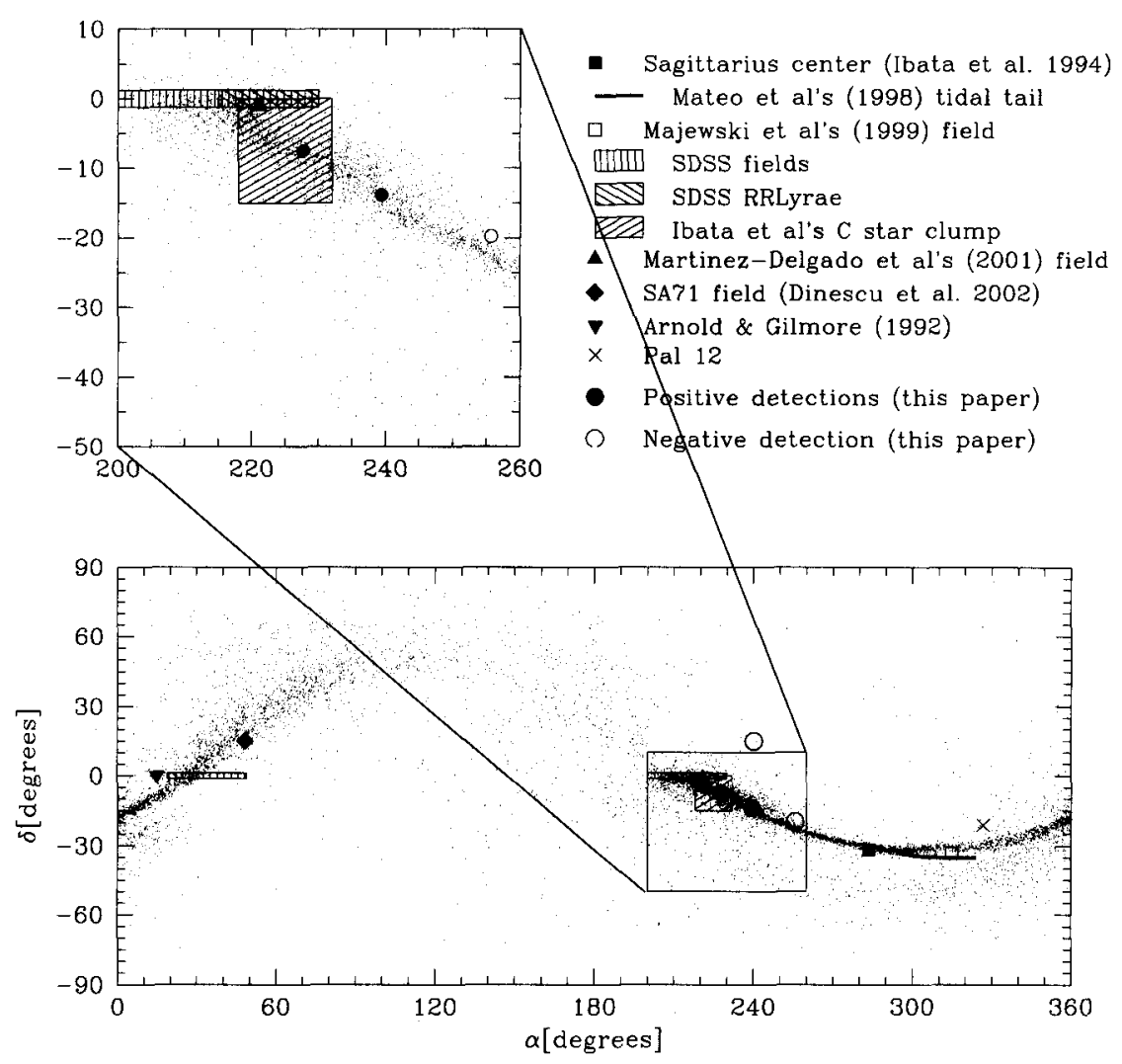

Figure 7. Dynamical model from the Sagittarius stream from Martínez-Delgado et al. (2003). The center of the galaxy, marked by a square, corresponds to the position of the M54 globular cluster. The positions of the globular clusters $\mathrm{Pal}$ 2, Pal 12, and Pal 14 are also marked. Other symbols correspond to several detections of the galaxy. See Martínez-Delgado et al. (2003) for references.

depends on the star formation rate (SFR) as a function of time. Determining the SFR is a complicated task, but things are made still more difficult because gas infall into and outflow from the intergalactic medium also play a quite fundamental role. Chemical enrichment laws are often assumed in astrophysical research, but they have only very recently obtained observationally. An example is the Fornax dSph galaxy (Pont et al. 2003). This galaxy is situated $138 \mathrm{kpc}$ from the Milky Way. At this distance it is possible i) to measure the metallicity of upper red giant branch (RGB) stars from the Ca II triplet and ii) to determine age of the same stars from a direct comparison with the isochrones of the corresponding metallicity. The latter (and perhaps the former) produces inaccurate 


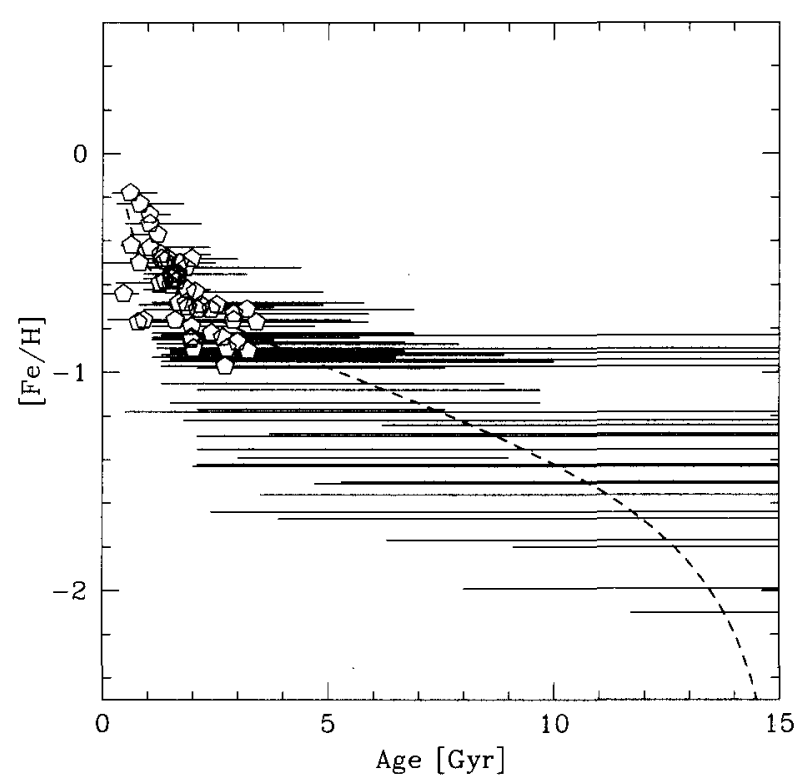

Figure 8. The metallicity law of the Fornax dwarf galaxy. Dots correspond to the age-metallicity pairs measured for the sample stars. The fit reproduces the metallicity law corresponding to the best solution for star formation, infall, and outflow scenario (Pont et al. 2003).

absolute scales. But the internal accuracy is high. Fig. 8 shows a metallicityage plot for the star sample observed in Fornax. The dot distribution can be matched to metallicity laws derived from several enrichment scenarios. The one shown corresponds to the best solution for infall and outflow rates, assuming a simple case of constant SFR (Pont et al. 2003).

\section{Quantitative determinations of star formation histories}

Galaxies evolve along two main paths: dynamically, including interaction with external systems, and through the process of the formation, evolution, and death of the stars within them. The latter has the following relevant effects on the galaxy: i) the evolution of gas content, ii) chemical enrichment, and iii) the formation of the stellar populations. The star formation history (SFH), is therefore fundamental to understanding the galaxy evolution process. We define the SFH as composed of two simpler functions: the SFR as a function of time and the chemical evolution law (CEL). The explicit determination of the SFH requires direct information about age and metallicity of the stars belonging to successive stellar populations. The color-magnitude diagram (CMD) provides this kind of information for large star samples and is the best tool for deriving the SFH. But for this information to be useful, the CMD must be deep and accurate, which is only reachable in the Local Group or its neighborhood. 


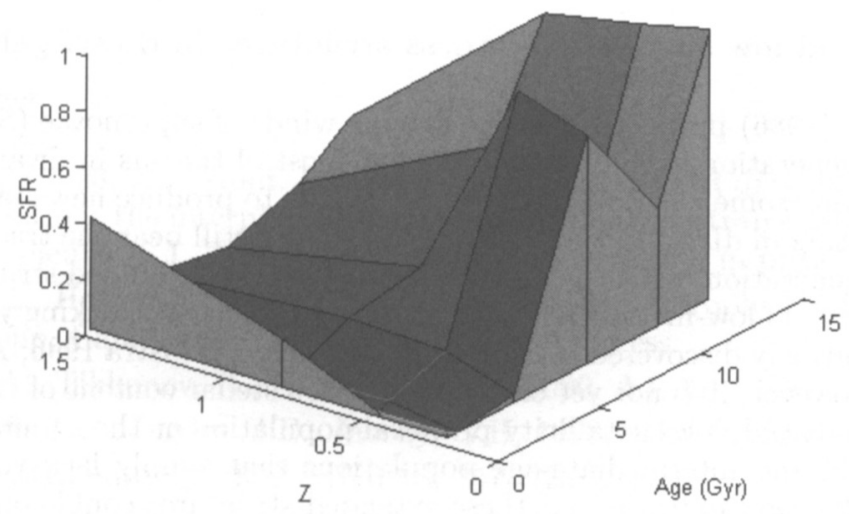

Figure 9. The population box of the dwarf galaxy Phoenix. Solutions of this kind, in which both the SFR and the CEL are simultaneously derived as a function of time, are possible for deep and accurate CMDs (Hidalgo et al. 2004). This result is preliminary.

Deriving a quantitative, accurate SFH is, in any case, complicated and requires a more-or-less sophisticated technique. The standard procedure involves three main ingredients: i) good data, from which a deep observational CMD, ideally reaching the oldest main-sequence turn-off stars, can be plotted; ii) a stellar evolution library providing colors and magnitudes of stars as a function of age, mass, and metallicity, and iii) a method for relating the number of stars populating different regions of the observational CMD with the density distribution of stars in the CMD as a function of age, mass, and metallicity, as predicted by the stellar evolution models. The latter will provide the SFH of the system. A review of the procedures is given by Aparicio (2003).

The CMD depth is fundamental if an SFH extending to the early epoch of galaxy evolution as a star-forming system is required. For CMDs showing only the RGB and the upper main sequence and blue loops, only an integrated estimate of the SFR is possible for ages older than a few hundred million years. In figure 6 of the review by Aparicio (2003), the SFRs of the galaxies are plotted for which, at the time of writing of that paper in 2001, a quantitative study of the CMD had been done using synthetic CMD techniques. In these cases the SFR was derived for the entire galaxy lifetime. Other, less accurate or less time extended SFRs are shown in figure 7 of the same paper.

With accurate enough data, the simultaneous derivation of the SFR and the CEL is possible, providing the full SFH as we have previously defined it. Fig. 9 shows an example of that for the dwarf galaxy Phoenix (Hidalgo, Aparicio, \& 
Martínez-Delgado 2004). A plot of this kind, in which the SFR and the CEL are plotted as a function of time, is customarily called a "population box" (Hodge $1989)$.

\section{Extended low surface brightness structures in dwarf galaxies}

Dekel \& Silk (1986) proposed that the driving winds of supernovae (SNe) of the first stellar generation should have swept out most of the gas in dwarf galaxies. Those retaining some gas would eventually be able to produce new stellar generations, resulting in dIrrs. In such cases, dIrrs should still bear the traces of their first stellar generation, which would be seen today as an extended structure populated by very old low-metallicity stars. Extended structures lacking young stars are being routinely discovered in dIrrs (e.g., Minniti, \& Zijlstra 1996; Aparicio et al. 2000). However, it is not yet clear whether the stellar content of these structures is a really old, low-metallicity primeval population or the superposition of successive old and intermediate-age populations that simply lack young stars. Recent results even indicate that these extended structures could contain a significant young population. Particularly interesting is the work by Komiyama et al. (2003) on the dwarf galaxy NGC 6822. This galaxy was known to have a gas component much more extended than the conspicuous optical component (Roberts 1972; de Blok \& Walker 2000). These very extended gas structures are common in gas-rich dwarf galaxies (see Roberts 1972 for several examples). Komiyama et al. (2003), from wide-field observations obtained with the Subaru Telescope, have obtained a map of young blue stars, as shown in their figure 4 . The distribution of young stars matches the extended gas component reasonably well. Even more, the young stars seem to trace a very low surface brightness spiral arm structure. The high surface brightness body of NGC 6822, which had been considered the only stellar component of the galaxy, turns out to be only its central component.

On another hand, dynamical models published by Mayer et al. (2001; see their figure 12) show that, evolving within a big galaxy potential well, high surface brightness galaxies (as dwarf irregulars) develop a low surface brightness spiral structure before becoming dwarf elliptical galaxies. This could indicate that galaxies like NGC 6822 could be in a transitional phase toward a dwarf elliptical morphology.

\section{Final considerations}

In this paper I have reviewed only a few relevant topics related to Local Group astrophysics. There are, however, several fields of astrophysical research, ranging from stellar astrophysics to cosmology, that could benefit from study of Local Group. In his book The Realm of Nebulae, Hubble (1936) pointed out that the study of individual galaxies in the Local Group was important because 1) they were "the nearest and most accessible examples of their particular types" and 2) they provided "a sample collection of nebulae, from which criteria can be derived for further exploration". Hubble's motivation continues to be valid today. 
Acknowledgments. I am grateful to Drs Gallart, Pont, Hidalgo, Rosenberg, Martínez-Delgado, and Gómez-Flechoso for fruitful discussions and comments that have largely improved this paper, and to Drs Pont, Zinn, Gallart, Hardy, and Winnick for having made the data of one of their plots available to me (Fig. 8 of this paper).

\section{References}

Aparicio, A. 2003, ASP Conf. Ser., Vol. nnn, Observed HR diagrams and stellar evolution: the interplay between observational constraints and theory, ed. T. Lejeune \& J. Fernandes, (San Francisco: ASP), in press

Aparicio, A., Herrero, A., Sánchez, F. 1998, Stellar Astrophysics for the Local Group, Cambridge: Cambridge University Press.

Aparicio, A., Tikhonov, N., Karachentsev, I. 2000, AJ, 119, 177

Armandroff, T.E., Davies, J.E., Jacoby, G.H. 1998, AJ, 116, 2287

Arnold, R., Gilmore, G. 1992, MNRAS, 257, 225 2000,

Chaboyer, B., Demarque, P., Sarajedini, A. 1996, ApJ, 459, 558

Corwin, H.G., de Vaucouleurs, G., de Vaucouleurs, A. 1985, Southern Galaxy Catalog, Austin: University Texas Press

de Blok, E., Walter, F. 2000, ApJ, 537, L95

Dekel, A., Silk, J. 1986, ApJ, 303, 39

Dinescu, D.I., et al. 2002, ApJ, 575, L67

Eggen, O.J., Lynden-Bell, D., Sandage, A. 1962, ApJ, 136, 748

Grebel, E.K. 1997, Rev. Mod. Astron., 10, 29

Grebel, E.K., Guhathakurta, P. 1999, ApJ, 511, L101

Hidalgo, S., Aparicio, A., Martínez-Delgado, D. 2004, in preparation

Hodge, P.W. 1989, ARA\&A, 27, 139

Hubble, E. 1936, The Realm of the Nebulae, New Haven: Yale University Press

Ibata, R.A., Gilmore, G. Irwin, M.J. 1994, Nature, 370, 194

Ibata, R., Lewis, G.F., Irwin, M., Totten, E., Quinn, T. 2001, ApJ, 551, 294

Irwin, M.J., Demers, S., Kunkel, W.E. 1990, AJ, 99, 191

Karachentsev, I. 1994, Astron. Astrophys. Trans., 6, 1

Karachentsev, I., Makarov, D. 2001, Ap, 44, 1

Komiyama, Y., Okamura, S., Yagi, M., et al. 2003, ApJ, 590, L17

Lavery, R.J. 1990, IAU circ. 5139

Layden, A., Smith, R.C., Storm, J. 1994, The Local Group: Comparative and Global Properties, Garching: ESO

Majewski, S.R., Siegel, M.H., Kunkel, W.E., Reid, I.N., Johnston, K.V., Thompson, I.B., Landolt, A.U., Palma, C. 1999, AJ, 118, 1707

Martínez-Delgado, D., Aparicio, A., Gómez-Flechoso, M.A., Carrera, R. 2001, ApJ, 549, L199

Martínez-Delgado, D., Gómez-Flechoso, M.A., Aparicio, A., Carrera, R. 2003, ApJ, in press 
Mateo, M. 1998, ARA\&A, 36, 435

Mateo, M., Olszewski, E.W., Morrison, H.L. 1998, ApJ, 508, L55

Mayer, L., Governato, F., Colpi, M., et al. 2001, ApJ, 559, 754

Minniti, D., Zijlstra, A.A. 1996, ApJ, 467, L13

Morrison, H., Harding, P., Hurley-Keller, D., Jacoby, G. 2003, ApJ, in press

Pont, F., Zinn, R., Gallart, C., Hardy, E., Winnick, R. 2003, AJ, in press

Roberts, M.S. 1972, in External Galaxies and Quasi-stellar Objects, IAU Symp. 44, ed. D.S. Evans, Reidel: Dordrecht, p. 12

Rosenberg, A., Saviane, I., Piotto, G., Aparicio, A. 1999, AJ, 118, 2306

Rosenberg, A., Saviane, I., Piotto, G., Aparicio, A. 2004, in preparation

Sandage, A. 1986, ApJ, 307,1

Searle, L., Zinn, R. 1978, ApJ, 225, 357

van den Bergh, S. 2000, The Galaxies of the Local Group, Cambridge: Cambridge University Press.

Whitelock P.A., Cannon, R.D. 1999, Stellar Content of the Local Group, I.A.U. Symp. 192, (San Francisco: ASP)

Whiting, A.B., Irwin, M.J., Hau, G.K.T. 1997, AJ, 114, 996

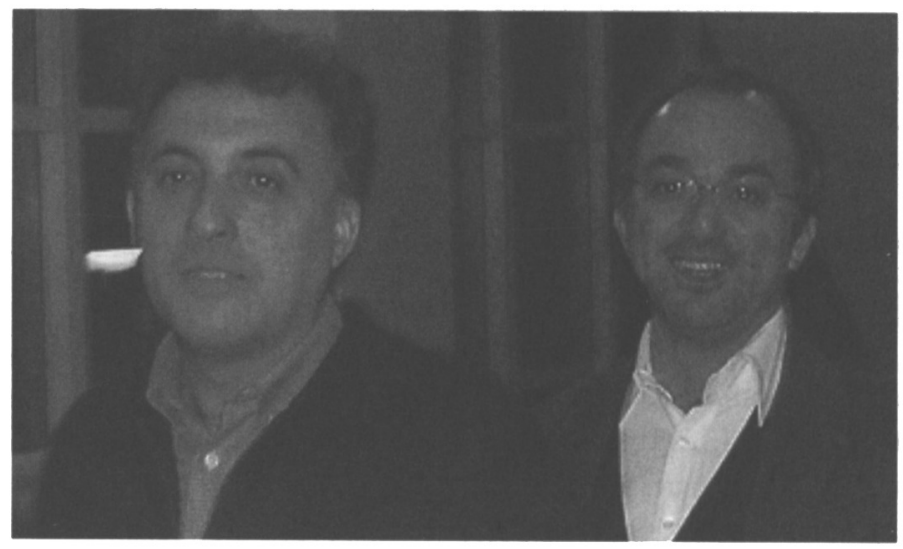

Antonio Aparicio and Guiseppe Bono 